\title{
From Differential Equations to the Construction of New Wavelet-Like Bases
}

\author{
Ildar Khalidov and Michael Unser, Fellow, IEEE
}

\begin{abstract}
In this paper, an approach is introduced based on differential operators to construct wavelet-like basis functions. Given a differential operator $L$ with rational transfer function, elementary building blocks are obtained that are shifted replicates of the Green's function of $\mathrm{L}$. It is shown that these can be used to specify a sequence of embedded spline spaces that admit a hierarchical exponential B-spline representation. The corresponding B-splines are entirely specified by their poles and zeros; they are compactly supported, have an explicit analytical form, and generate multiresolution Riesz bases. Moreover, they satisfy generalized refinement equations with a scale-dependent filter and lead to a representation that is dense in $L_{2}$. This allows us to specify a corresponding family of semi-orthogonal exponential spline wavelets, which provides a major extension of earlier polynomial spline constructions. These wavelets are completely characterized, and it is proven that they satisfy the following remarkable properties: 1) they are orthogonal across scales and generate Riesz bases at each resolution level; 2) they yield unconditional bases of $L_{2}$-either compactly supported (B-spline-type) or with exponential decay (orthogonal or dual-type); 3) they have $N$ vanishing exponential moments, where $N$ is the order of the differential operator; 4) they behave like multiresolution versions of the operator $L$ from which they are derived; and 5) their order of approximation is $(N-M)$, where $N$ and $M$ give the number of poles and zeros, respectively. Last but not least, the new wavelet-like decompositions are as computationally efficient as the classical ones. They are computed using an adapted version of Mallat's filter bank algorithm, where the filters depend on the decomposition level.
\end{abstract}

Index Terms-Continuous-time signal processing, differential operators, Green's functions, multiresolution analysis, multiresolution approximation, splines, wavelets.

\section{INTRODUCTION}

$\mathbf{I}$ $\mathrm{N}$ recent years, the wavelet transform has emerged as a powerful tool for performing multiresolution signal analysis and processing [1]-[3]. Wavelets have led to a multitude of applications with a significant impact on image compression, communications, and on other areas of applied mathematics. There has also been an intense activity in wavelet design leading to the construction of a large variety of wavelet bases, the most prominent ones being tailored to special requirements such as orthogonality and short support [4], high number of vanishing moments [5], symmetry and regularity [6], explicit analytical

Manuscript received August 12, 2004; revised April 17, 2005. This work was supported in part by the Swiss National Science Foundation under Grant 200020-101821. The associate editor coordinating the review of this manuscript and approving it for publication was Prof. Karim Drouiche.

The authors are with the Biomedical Imaging Group, Ecole Polytechnique Fédérale de Lausanne (EPFL), CH-1015 Lausanne, Switzerland (e-mail: ildar.khalidov@epfl.ch; michael.unser@epfl.ch).

Digital Object Identifier 10.1109/TSP.2006.870544 form [7]-[9], near-optimal time-frequency localization [10], to cite but a few.

One of the key mathematical properties of wavelets is that they behave like multiscale differentiators [1], i.e., the wavelet coefficients of a signal are the samples of the $N$ th-order derivative of smoothed versions of it. Thus, there is a correspondence between a wavelet with $N$ vanishing moments and the differentiation operator $\mathrm{D}^{N}$. One of the questions that motivated this work is: What happens if we consider an arbitrary linear differential operator $\mathrm{L}$ instead of $\mathrm{D}^{N}$ ? Will we be able to construct wavelets that qualitatively behave like L? In this paper, we show that this is indeed possible for differential operators with arbitrary rational transfer functions.

The derivative-like behavior of wavelets was investigated in some depth in [11]. Specifically, it was shown that it is the regular component of the scaling function (i.e., the polynomial B-spline that lies hidden within) that induces this property. Mathematically, this behavior turns out to be intimately linked to the property that the polynomial B-spline of order $N$ is a localized version (i.e., a linear combination of shifted replicates) of the Green's function of the operator $\mathrm{D}^{N}$. Thus, a possible way to induce a modified wavelet behavior is to consider generators that are localized versions of the Green's functions of more general differential operators. In the case of ordinary differential operators, the exponential B-splines are natural candidates [12]. In the cardinal setting, it has been shown recently that these B-splines share all important properties of their polynomial counterparts: they are compactly supported, they generate Riesz bases, and they satisfy some general multiresolution-like embedding properties [13], [14]. In this paper, we go one step further and use these functions to specify an extended family of exponential spline wavelets that satisfy some interesting mathematical properties. While we believe that our proposal to construct wavelets based on operators with rational transfer function is novel, there is a theoretical connection with earlier work on nonstationary wavelets [15]-[20]. For instance, the present construction falls within the generalized multiresolution framework of de Boor, DeVore and Ron [16]. These authors even briefly considered exponential splines as an illustrative example of their general scheme ([16, sec. 6]); they established the existence of such multiresolution bases of $L_{2}$, but they did not go into much practical details beyond this. Also relevant is the work of Lyche and Schumaker [19], who construct general L-spline wavelets with nonuniform knots on the interval, starting with a time-varying differential operator. Finally, there is a link between exponential spline wavelets and some nonstationary subdivision schemes that preserve exponential polynomials [21]. We note, however, that 
these only correspond to the low-pass synthesis part of the wavelet algorithm and that the filters are interpolating, which is typically not the case here.

This paper is organized as follows. In Section II, we illustrate our wavelet-construction method by producing an extension of the Haar transform that is matched to the first-order differential operator $(\mathrm{D}-\alpha \mathrm{I})$ with parameter $\alpha \in \mathbb{C}$. In Section III, we consider the general case of a differential operator $\mathrm{L}$ and introduce the corresponding exponential B-splines as space-generating functions. We specify the embedding properties of the exponential B-spline spaces and give a new result on their approximation properties that guarantees that the representation is dense in $L_{2}$. In Section IV, we use these functions to construct orthonormal and semi-orthogonal wavelets and characterize the corresponding filters. This provides the generalization of the polynomial spline wavelet family [9] for a much larger class of splines. In Section V, we identify the key mathematical properties of the exponential B-spline wavelets. Finally, we discuss the computational aspects of the introduced wavelet transform and consider specific examples.

\section{EXPONENTIAL EXTENSION OF THE HAAR SYSTEM}

To introduce our new wavelet concept, we start with a simple illustrative example and show how piecewise exponentials can be used to construct an extended version of the Haar transform.

\section{A. E-Spline Multiresolution: First-Order Case}

Consider the first-order linear differential operator $\mathrm{L}=\mathrm{D}-$ $\alpha \mathrm{I}: \mathrm{L} f=f^{\prime}-\alpha f, \alpha \in \mathbb{C}$. As is well known from linear differential equation theory, the Green's function for this operator is $\rho_{\alpha}(t)=e^{\alpha t} \cdot u(t)$, where $u(t)$ is the step function. We recall that the Green's function $\rho$ is causal and satisfies $\mathrm{L} \rho=\delta$, where $\delta$ denotes the Dirac impulse; it is simply the impulse response of the causal inverse operator $\mathrm{H}=\mathrm{L}^{-1}$.

By definition, the exponential spline $s(t)$ associated with the operator $\mathrm{L}$ is a function such that

$$
\mathrm{L}\{s\}(t)=\sum_{k \in \mathbb{Z}} a[k] \delta\left(t-t_{k}\right)
$$

where the $a[k]$ 's are arbitrary coefficients, and where the $t_{k}$ 's are called the knots of the spline. In our case where L is a firstorder differential operator, the spline $s(t)$, as defined above, is discontinuous at these points. We can integrate this equation by applying the inverse operator $\mathrm{H}=(\mathrm{D}-\alpha \mathrm{I})^{-1}$ to this relation, which yields

$$
s(t)=\sum_{k \in \mathbb{Z}} a[k] \rho_{\alpha}\left(t-t_{k}\right)+p_{\alpha}(t)
$$

where the additional term $p_{\alpha}(t)=p_{0} e^{\alpha t}$, with $p_{0} \in \mathbb{C}$, is a solution of the homogeneous equation $\mathrm{L} p_{\alpha}=0$ to be chosen so that $s(t)$ satisfies specific boundary conditions.

To be able to apply fast filtering algorithms, we restrict ourselves to the case where the knots are equally spaced; i.e., $t_{k}=$ $T k, k \in \mathbb{Z}$, where $T$ is the interval between two knots. The corresponding spline space $V_{T}=\operatorname{span}\left\{\rho_{\alpha}(\cdot-T k)\right\}$ is in this case $T$-shift invariant. One can also omit $p_{\alpha}$, the element of the null

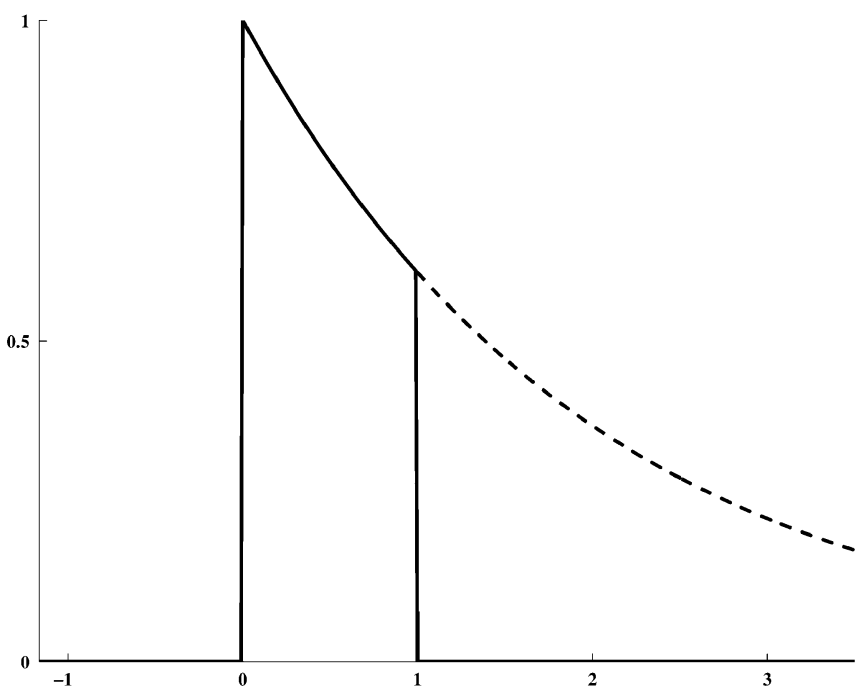

Fig. 1. Localization of the Green's function, $T=1$.

space of L in (1), because it can be expressed through the shifts of the Green's function [13].

While the representation of a spline in terms of shifted Green's functions $\rho_{\alpha}(t-T k)$ is attractive conceptually, it has the disadvantage of involving basis functions that are not compactly supported. The key idea, which is in the foundation of the present paper, is that one can construct an equivalent set of compactly supported basis functions by taking a suitable linear combination of basis functions. It is not difficult to see that the shortest possible functions in $V_{T}$ take the form

$$
\beta_{\alpha, T}(t)=\rho_{\alpha}(t)-e^{\alpha T} \rho_{\alpha}(t-T) .
$$

This function, which is compactly supported in $[0, T]$, is the exponential B-spline of order one. In effect, the Green's function is truncated by substracting its weighted and shifted version (see Fig. 1).

The $T$-integer-shifted B-splines $\left\{\beta_{\alpha, T}(t-T k)\right\}_{k \in \mathbb{Z}}$ are obviously orthogonal to each other, and they form a Riesz basis. Conversely, it is also possible to invert (2) and to express the Green's function as the linear combination of B-splines

$$
\rho_{\alpha}(t)=\sum_{k=0}^{\infty} e^{\alpha k T} \beta_{\alpha, T}(t-T k) .
$$

The above Green's function reproduction formula, which is easily visualized graphically, implies that all $T$-shifts of the Green's function $\rho_{\alpha}$ belong to $\operatorname{span}\left\{\beta_{\alpha, T}(\cdot-T k)\right\}$, and thus

$$
V_{T}=\operatorname{span}\left\{\beta_{\alpha, T}(\cdot-T k)\right\} \text {. }
$$

The Green's function itself does not depend on the step size $T$. Thus, given a fixed parameter $\alpha$, we can construct the Green's function $\rho_{\alpha}$ and build $V_{T}$ as a span of $T$-shifts of $\rho_{\alpha}$. On the contrary, the B-splines $\beta_{\alpha, T}(t)$ depend on the scale imposed by $T$, and this is the price paid for their compact support. Moreover, if we compare the B-spline to a standard scaling function, we can clearly see that the relation between the scales $T$ and $2 T$ is no longer a dilation (see Fig. 2). 

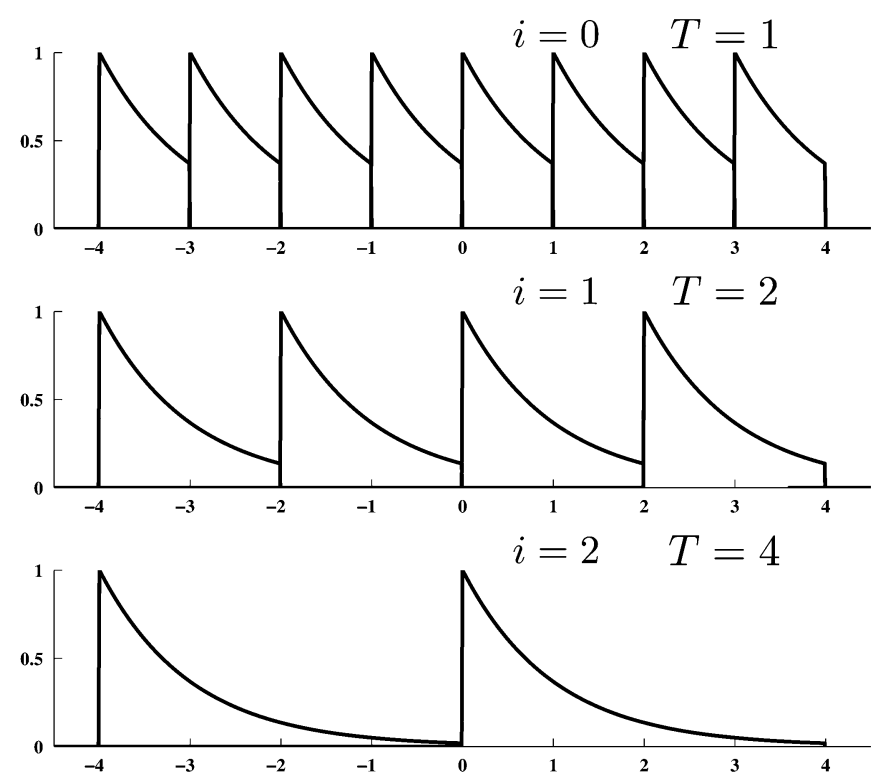

Fig. 2. Multiresolution analysis: E-spline representation.

So far, we have constructed the spaces $V_{T}$. The Green's function representation makes the inclusion $V_{2 T} \subset V_{T}$ obvious, as illustrated in Fig. 3. It is then a natural step to attempt the construction of a multiresolution-like structure using B-splines at a dyadic scale $T=2^{i}$. For this purpose, we localize the Green's functions and consider B-splines as basis functions (see Fig. 2). What we get is not a multiresolution analysis in the classical sense because it is not dilation that links the B-splines at different scales. Also, as soon as $\rho_{\alpha} \in L_{2}$ belongs to each space $V_{2^{i}}$ (i.e., when $\operatorname{Re}\{\alpha\}<0$ ), the intersection $\cap_{i=-\infty}^{\infty} V_{(i)}$ is not empty. In this case, this happens because the Green's function is square-integrable. This means that the sum in the wavelet-space decomposition of $L_{2}$ must start from a finite scale and include the corresponding low-pass space. One should mention, however, that in practical applications one always keeps low-pass data at the coarsest scale, which makes the empty-intersection property irrelevant.

From now on, we denote $V_{(i)}$ the space $V_{T}$ with $T=2^{i}, c_{i}=$ $\left\|\beta_{\alpha, 2^{i}}\right\|_{L_{2}}$, and $\varphi_{i}(t)=\left(1 / c_{i}\right) \beta_{\alpha, 2^{i}}(t)$, the corresponding normalized generating function. As we see from the Green's function representation of these spaces, $V_{(i+1)}$ is a subset of $V_{(i)}$. This means that we can expand $\varphi_{i+1} \in V_{(i+1)}$ in $\left\{\varphi_{i}(\cdot-\right.$ $\left.\left.2^{i} k\right)\right\}_{k \in \mathbb{Z}}$. Specifically, we have that

$$
\varphi_{i+1}(t)=\frac{c_{i}}{c_{i+1}}\left(\varphi_{i}(t)+e^{2^{i} \alpha} \varphi_{i}\left(t-2^{i}\right)\right),
$$

which points out a fundamental difference with conventional wavelet theory: The present filter coefficients exhibit a scale dependence. The Fourier expression for the refinement filter is

$$
H_{\alpha, i}\left(e^{j 2^{i} \omega}\right)=\frac{\hat{\varphi}_{i+1}(\omega)}{\hat{\varphi}_{i}(\omega)}=\frac{c_{i}}{c_{i+1}} \cdot\left(1+e^{2^{i}(\alpha-j \omega)}\right)
$$

which is clearly $\left(2^{-i} \cdot 2 \pi\right)$-periodic in $\omega$.

The next step is to examine the orthogonal complement $W_{(i+1)}$ such that $V_{(i+1)} \oplus W_{(i+1)}=V_{(i)}$, as it is done in classical wavelet construction. It is not difficult to see that
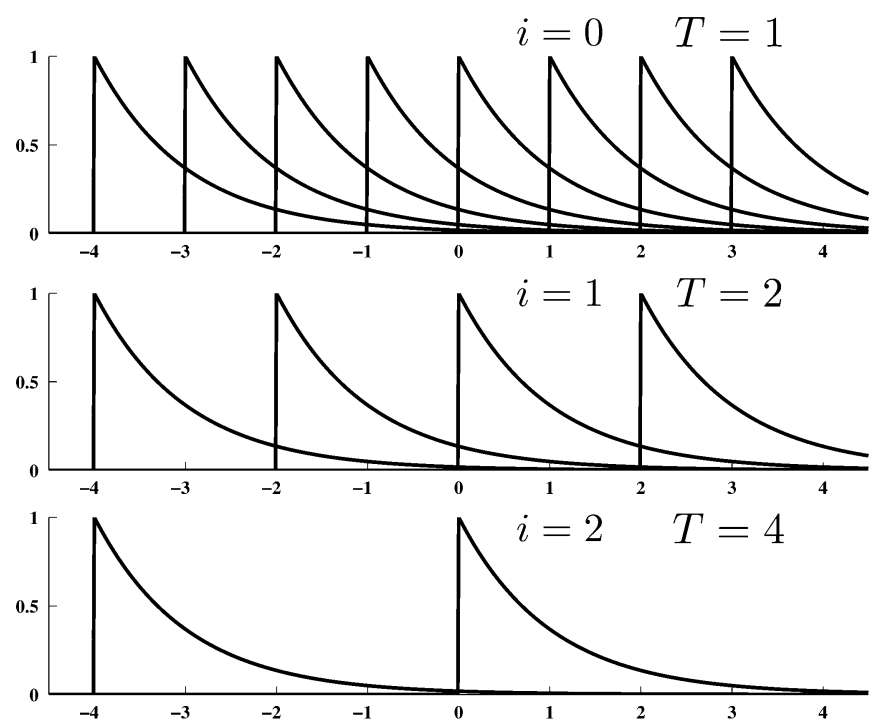

Fig. 3. Toward a multiresolution analysis: Green's function representation of spline spaces. The basis of $V_{2 T}$ contains every other element of the basis of $V_{T}$.
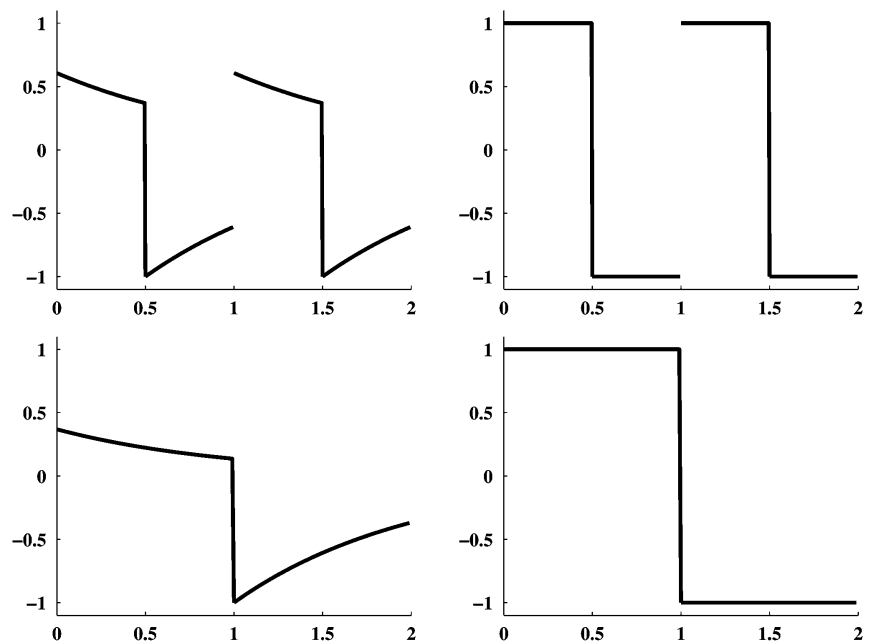

Fig. 4. Left: First-order E-spline wavelet $\psi_{i}$ at different scales. Right: Haar wavelet at different scales.

the function $\psi_{i+1}(t)=\left(c_{i} / c_{i+1}\right)\left(e^{2^{i} \alpha^{*}} \varphi_{i}(t)-\varphi_{i}\left(t-2^{i}\right)\right)$ is a generator of $W_{(i+1)}$. It is clearly included in $V_{(i)}$ and is orthogonal to $\varphi_{i+1}$, as justified by

$$
\begin{aligned}
\left\langle\varphi_{i+1}, \psi_{i+1}\right\rangle=\frac{c_{i}^{2}}{c_{i+1}^{2}} e^{2^{i} \alpha} \int_{0}^{2^{i}}\left|e^{2 \alpha t}\right| \mathrm{d} t & \\
& -\frac{c_{i}^{2}}{c_{i+1}^{2}} \int_{2^{i}}^{2^{i+1}} e^{2^{i} \alpha} \cdot\left|e^{2 \alpha\left(t-2^{i}\right)}\right| \mathrm{d} t=0 .
\end{aligned}
$$

We show this wavelet in Fig. 4. We observe that the Haar wavelet corresponds to $\alpha=0$.

There are several important properties that can be seen with this first E-spline wavelet. First, its integral is not necessarily zero, which means that the corresponding filter is not necessarily high pass. Second, the wavelets (and, consequently, their spectra) at different scales are not dilated replica of each other. For instance, when $\alpha=j \omega_{0}$, the wavelet is complex of constant amplitude and its spectrum is shifted by $\omega_{0}$ as compared to the 
Haar case. Finally, we can prove that the extended Haar multiresolution is dense in $L_{2}$ (cf. Section III-B). This is the final ingredient that is required to have a wavelet-like basis of $L_{2}$.

\section{E-SPLINES}

In this section, we generalize the previous construction by introducing E-splines associated with higher-order operators and by defining the corresponding multiresolution analysis of $L_{2}$. We then consider the approximation properties of the constructed multiresolution representation and prove that it is dense in $L_{2}$.

\section{A. E-Spline Multiresolution}

We start with the specification of a linear differential system. This system takes an input function $x(t)$ and produces an output $y(t)$; its behavior is generally described by

$$
\begin{aligned}
\mathrm{D}^{N} y+a_{N-1} \mathrm{D}^{N-1} y+ & \cdots+a_{0} y \\
& =\mathrm{D}^{M} x+b_{M-1} \mathrm{D}^{M-1} x+\cdots+b_{0} x
\end{aligned}
$$

with $M<N$. This equation can also be written in the equivalent operator form $\mathrm{L}\{y\}=x$. To obtain the transfer function of $\mathrm{L}$, we take the Laplace transform of both sides and solve for $X(s) / Y(s)$, which yields

$$
L_{\vec{\alpha}, \vec{\gamma}}(s)=\frac{\Pi_{n=1}^{N}\left(s-\alpha_{n}\right)}{\Pi_{m=1}^{M}\left(s-\gamma_{m}\right)}
$$

with parameter vectors $\vec{\alpha}=\left(\alpha_{1}, \ldots, \alpha_{N}\right)$ and $\vec{\gamma}=$ $\left(\gamma_{1}, \ldots, \gamma_{M}\right)$, where $\left\{\alpha_{n}\right\}_{n=1}^{N}$ and $\left\{\gamma_{m}\right\}_{m=1}^{M}$ are the roots of the polynomials $s^{N}+a_{N-1} s^{N-1}+\cdots+a_{0}$ and $s^{M}+b_{M-1} s^{M-1}+\cdots+b_{0}$, respectively. The Green's function of the system is given by

$$
\rho_{\vec{\alpha}, \vec{\gamma}}(t)=\mathcal{L}^{-1}\left\{\frac{\Pi_{m=1}^{M}\left(\cdot-\gamma_{m}\right)}{\Pi_{n=1}^{N}\left(\cdot-\alpha_{n}\right)}\right\}(t)
$$

which can be determined by explicitly computing the inverse Laplace transform. We will therefore refer to the spline-defining parameters, $\left\{\alpha_{n}\right\}_{n=1}^{N}$ and $\left\{\gamma_{m}\right\}_{m=1}^{M}$, as the poles and the zeros, respectively.

A generalized E-spline with vector of poles $\vec{\alpha}$, vector of zeros $\vec{\gamma}$ and knots $-\infty<\cdots<t_{k}<t_{k+1}<\cdots<+\infty$ is a function of the form

$$
s(t)=\sum_{k \in \mathbb{Z}} a[k] \rho_{\vec{\alpha}, \vec{\gamma}}\left(t-t_{k}\right)+p_{\vec{\alpha}, \vec{\gamma}}(t)
$$

where $p_{\vec{\alpha}, \vec{\gamma}}$ is a linear combination of exponential polynomials from the null space $\mathcal{N}_{\vec{\alpha}}$ of the operator $L_{\vec{\alpha}, \vec{\gamma}}$. To make the notation simpler, from now on, we will omit the indexes $\vec{\alpha}, \vec{\gamma}$.

As in our illustrative example, we now consider exponential splines on a uniform grid with knots $t_{k}=T k, k \in \mathbb{Z}$. In this case [14], all null-space elements $p \in \mathcal{N}_{\vec{\alpha}}$ are reproduced by $\{\rho(\cdot-$ $T k)\}_{k \in \mathbb{Z}}$. Consequently, from the definition of an E-spline, the shifted versions of the Green's functions form a basis of the space of generalized exponential splines.

Now, we are interested in a localized basis function. If $\vec{\gamma}$ is empty, the Green's function is a convolution of first-order ones, and we use the composition of first-order localization operators (which corresponds to the convolution of their impulse responses) to localize it, as in

$$
\Delta_{\vec{\alpha}, T}=\Delta_{\left(\alpha_{1}, \alpha_{2}, \ldots, \alpha_{n}\right), T}=T\left(\Delta_{\left(\alpha_{1}\right), T} \ldots \Delta_{\left(\alpha_{n}\right), T}\right)
$$

where $\Delta_{\left(\alpha_{i}\right), T} f(t)=(1 / T)\left(f(t)-e^{\alpha_{i} T} f(t-T)\right)$. It can be further seen [14] that the same localization operator $\Delta_{\vec{\alpha}, T}$ can be used to localize $\rho_{\vec{\alpha}, \vec{\gamma}}$ for arbitrary $\vec{\gamma}$, as long as $M<N$.

The function $\beta_{T}(t)=\beta_{\vec{\alpha}, \vec{\gamma}, T}(t)=\Delta_{\vec{\alpha}, T} \rho(t)$ is called an exponential B-spline; it is supported in [0,TN). Its Fourier transform is given by

$$
\hat{\beta}_{T}(\omega)=\frac{1}{T^{N-1}} \prod_{k=1}^{N} \frac{1-e^{T\left(\alpha_{k}-j \omega\right)}}{\left(j \omega-\alpha_{k}\right)} \prod_{l=1}^{M}\left(j \omega-\gamma_{l}\right) .
$$

We assume the stability condition $\alpha_{l}-\alpha_{m} \neq 2 \pi k j / T$ for all distinct pure imaginary roots $\alpha_{l}, \alpha_{m}$. The $T$-integer shifts of the B-spline then form a Riesz basis [14, Th. 1], i.e., they provide a stable signal representation. In addition, one can show that the Green's function can be reconstructed as

$$
\rho(t)=\sum_{k=0}^{+\infty} p_{T}[k] \beta_{T}(t-T k)
$$

where $p_{T}[k]$ are some suitable weights [14]. This reproduction formula ensures the completeness of $\left\{\beta_{T}(\cdot-T k)\right\}_{k \in \mathbb{Z}}$ in the space spanned by the shifted Green's functions. Thus, the exponential B-splines form a stable and complete basis of this space.

To define a corresponding multiresolution analysis, we focus on the dyadic scales $T=2^{i}$. The $V_{(i)}$ 's are the subspaces of $L_{2}$ spanned by $2^{i}$-shifts of the Green's function

$$
V_{(i)}=\left\{s_{i}=\sum_{k \in \mathbb{Z}} c_{k} \rho\left(\cdot-2^{i} k\right)\right\} \cap L_{2}
$$

where the $c_{k}$ 's are arbitrary coefficients.

Due to the equivalence provided by the localization process and by the Green's function reproduction formula, we can also write

$$
V_{(i)}=\left\{s_{i}(t)=\sum_{k \in \mathbb{Z}} c_{k} \varphi_{i}\left(t-2^{i} k\right): c \in l_{2}\right\}
$$

where $\varphi_{i}(t)$ is the normalized scaling function $\varphi_{i}(t)=$ $\beta_{2^{i}}(t) /\left\|\beta_{2^{i}}\right\|_{L_{2}}$.

We give in Fig. 5 a diagram that summarizes the construction of the exponential-spline spaces. The space $V_{(i)}$ is included in $V_{(i-1)}$ by construction. Moreover, as we shall see in the corollary to Theorem 1, the approximation error in $V_{(i)}$ goes to zero as $i \rightarrow-\infty$. We therefore have a ladder of spaces satisfying $\bar{U} V_{(i)}=L_{2}$. However, in contrast with the common definition of a multiresolution analysis, the scaling function whose shifts form a basis of $V_{(i)}$ is not a dilated version of $\varphi_{0}$ if $i \neq 0$. Instead, at each scale $i$, we have a specific generating function $\varphi_{i}$. Similarly to the first-order case, the causal Green's function $\rho$ belongs to all spaces $V_{(i)}$ if $\operatorname{Re}\left\{\alpha_{l}\right\}<0, l=1, \ldots, N$; else, their intersection is empty as is usually expected (i.e., $\cap V_{(i)}=$ $\emptyset)$. 


$$
L V_{T}=\operatorname{span}\{\delta(\cdot-T k)\}
$$

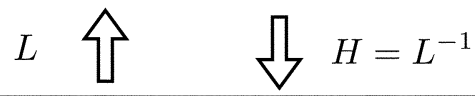

$V_{T}=\operatorname{span}\{\rho(\cdot-T k)\}$

(Green function reproduction)

$\Delta_{T}^{-1} \widehat{\Downarrow} \Delta_{T}$ (localization)

$$
V_{T}=\operatorname{span}\left\{\beta_{T}(\cdot-T k)\right\}
$$

Fig. 5. Construction of exponential-spline spaces.

\section{B. Order of Approximation}

To measure the quality of the E-spline approximation of a signal $f$, we estimate the asymptotic behavior of the approximation error as the scale $T=2^{i}$ goes to zero (or, equivalently, as $i \rightarrow-\infty$ ). There are results from basic spline theory, applicable to the case where $\vec{\gamma}$ is empty, that imply that the error should decay like $T^{N}$ as $T \rightarrow 0$ [12], [22]. An exact asymptotic formula is given in [13, Th. 2]. Here, we extend this result for the general rational case $(M<N)$.

Theorem 1: Let $L_{\vec{\alpha}, \vec{\gamma}}$ be a defining operator for a linear differential system with $\operatorname{dim}(\vec{\alpha})=N, \operatorname{dim}(\vec{\gamma})=M$. Let $f \in L_{2}$ be a function such that $\mathrm{D}^{N} f \in L_{2}$, and let $P_{T}$ denote the orthogonal projector into the exponential B-spline space $V_{T}$. Then, we have the following asymptotic formula for the approximation error as $T \rightarrow 0$ :

$$
\left\|f-P_{T} f\right\|_{L_{2}}=C_{N, M} \cdot T^{N-M} \cdot\left\|L_{\vec{\alpha}, \vec{\gamma}} f\right\|_{L_{2}}
$$

where $C_{N, M}=\left(\sqrt{2 \zeta(2(N-M))} /(2 \pi)^{N-M}\right)$ with $\zeta(s)=$ $\sum_{k=1}^{\infty} 1 / k^{s}$ (Riemann's zeta function).

The proof of this theorem is quite technical and is given in Appendix A. The following corollary ensures that the approximation order tends to zero as $T \rightarrow 0$ for any $f \in L_{2}$, as stated in the previous subsection.

Corollary 1: For any function $f \in L_{2}$ we have $\left\|f-P_{T} f\right\| \rightarrow$ 0 as $T \rightarrow 0$.

Proof: We use the fact that the Sobolev space $W_{2}^{N}$ is dense in $L_{2}$. Specifically, we estimate the error from above as $\| f-$ $P_{T} f\|\leq\| f-f_{s}\|+\| f_{s}-P_{T} f_{s}\|+\| P_{T}\left(f_{s}-f\right)\|\leq 2\| f-f_{s} \|+$ $\left\|f_{s}-P_{T} f_{s}\right\|$, where $f_{s} \in W_{2}^{N}$ can be chosen to be arbitrarily close to $f$.

Theorem 1 is a result for the approximation of smooth functions. For rougher functions whose Sobolev degree of smoothness is $r<N-M$, we expect that the error will only decay like $O\left(T^{r}\right)$. A proof for the standard wavelet case can be found in [23].

\section{Multiresolution Basis Functions}

So far, we have constructed the spaces $V_{(i)}$ spanned by the $2^{i}$-shifts of the Green's function and have given an equivalent representation using compactly supported B-splines. In this section, we construct orthonormal and dual basis-generating functions for $V_{(i)}$, as well as the corresponding wavelets.

\section{A. E-Spline Scaling Functions}

We start with a proposition that helps us construct Riesz bases in $V_{(i)}$. First, we define

$$
a_{i}[k]=\left\langle\varphi_{i}(\cdot), \varphi_{i}\left(\cdot-2^{i} k\right)\right\rangle
$$

which is the Gram sequence-or autocorrelation-of the basis $\left\{\varphi_{i}\left(t-2^{i} k\right)\right\}_{k \in \mathbb{Z}}$. We then have that $A_{i}\left(e^{j 2^{i} \omega}\right)=$ $\sum_{k \in \mathbb{Z}} a_{i}[k] e^{-j 2^{i} \omega k}=2^{-i} \sum_{k \in \mathbb{Z}}\left|\hat{\varphi}_{i}\left(\omega+2 \pi k / 2^{i}\right)\right|^{2}$, where $A_{i}(z)$ is the $z$-transform of $a_{i}[k] . A_{i}(z)$ is also referred to as the autocorrelation filter.

Proposition 1: Let $\varphi_{i}$ be an exponential B-spline at scale $T=2^{i}$, with exponential parameter vector $\vec{\alpha}$ such that $\alpha_{l}-$ $\alpha_{m} \neq j 2 \pi k / 2^{i}, k \in \mathbb{Z}$ for all distinct purely imaginary roots $\alpha_{l}, \alpha_{m}$. Then $\left\{\phi_{i}\left(\cdot-2^{i} k\right)\right\}_{k \in \mathbb{Z}}$, with

$$
\phi_{i}(t)=\sum_{k \in \mathbb{Z}} p_{i}(k) \varphi_{i}\left(t-2^{i} k\right)
$$

is a Riesz basis of $V_{(i)}$ if and only if $0<c_{1} \leq\left|P_{i}\left(e^{j \omega}\right)\right| \leq c_{2}<$ $\infty$.

Proof: We know that, if $\vec{\alpha}$ satisfies the conditions of the theorem, then $\left\{\varphi_{i}\left(\cdot-2^{i} k\right)\right\}_{k \in \mathbb{Z}}$ is a Riesz basis of $V_{(i)}[14$, Th. 1]. Considering the autocorrelation filter for $\phi_{i}$, we get

$$
\begin{aligned}
& \frac{1}{2^{i}} \sum_{k \in \mathbb{Z}}\left|\hat{\phi}_{i}\left(\omega+\frac{2 \pi k}{2^{i}}\right)\right|^{2} \\
& \quad=\frac{1}{2^{i}} \sum_{k \in \mathbb{Z}}\left|P_{i}\left(e^{j 2^{i} \omega}\right)\right|^{2} \cdot\left|\hat{\varphi}_{i}\left(\omega+\frac{2 \pi k}{2^{i}}\right)\right|^{2} \\
& \quad=\left|P_{i}\left(e^{j 2^{i} \omega}\right)\right|^{2} A_{i}\left(e^{j 2^{i} \omega}\right) .
\end{aligned}
$$

Thus, the left-hand side expression is positive and bounded if and only if $\left|P_{i}\left(e^{j \omega}\right)\right|$ is positive and bounded.

The compactly supported basis $\left\{\varphi_{i}\left(\cdot-2^{i} k\right)\right\}_{k \in \mathbb{Z}}$ that we constructed in the previous section is generally not orthogonal if the order of the exponential B-spline is greater than 1 . To construct a dual basis $\left\{\varphi_{i}\left(\cdot-2^{i} k\right)\right\}$ of the same space $V_{(i)}$, we write in the Fourier domain the condition of biorthonormality between $\left\{\stackrel{\circ}{\varphi}_{i}\left(\cdot-2^{i} k\right)\right\}$ and $\left\{\varphi_{i}\left(\cdot-2^{i} k\right)\right\}$, from which we deduce that

$$
\hat{\grave{\varphi}}_{i}(\omega)=\frac{\hat{\varphi}_{i}(\omega)}{A_{i}\left(e^{j 2^{i} \omega}\right)} .
$$

The dual basis is useful to project an arbitrary signal $f \in L_{2}$ onto $V_{(i)}$. This least-squares approximation is computed via the projection formula

$$
P_{i} f(x)=\sum_{k}\left\langle f(\cdot), \stackrel{\circ}{\varphi}_{i}\left(\cdot-2^{i} k\right)\right\rangle \varphi_{i}\left(\cdot-2^{i} k\right) .
$$


To build an orthonormal basis $\left\{\varphi_{o, i}\left(\cdot-2^{i} k\right)\right\}_{k \in \mathbb{Z}}$, we orthonormalize $\hat{\varphi}_{i}(\omega)$ in the Fourier domain, which yields

$$
\hat{\varphi}_{o, i}(\omega)=\frac{\hat{\varphi}_{i}(\omega)}{\sqrt{A_{i}\left(e^{j 2^{i} \omega}\right)}} .
$$

Since the weighting functions $\left(A_{i}\left(e^{j 2^{i} \omega}\right)\right)^{-1}$ and $\left(A_{i}\left(e^{j 2^{i} \omega}\right)\right)^{-(1 / 2)}$ are bounded from above and are nonvanishing, we can invoke Proposition 1 which ensures that the constructed dual and orthonormal bases are Riesz bases as well.

As noticed before, $V_{(i+1)} \subset V_{(i)}$. In particular, $\varphi_{i+1}$ can be decomposed in the basis of $V_{(i)}$, which gives us the scaling relation

$$
\varphi_{i+1}(t)=\sum_{k} h_{i}[k] \varphi_{i}\left(t-2^{i} k\right) .
$$

Taking the Fourier equivalent of this formula and plugging it in (3), we find that

$$
H_{i}\left(e^{j 2^{i} \omega}\right)=\frac{\hat{\varphi}_{i+1}(\omega)}{\hat{\varphi}_{i}(\omega)}=2 \frac{c_{i}}{c_{i+1}} \cdot \prod_{k=1}^{N} \frac{1+e^{2^{i}\left(\alpha_{k}-j \omega\right)}}{2}
$$

where $c_{i}=\left\|\beta_{i}\right\|_{L_{2}}$ is a normalizing constant.

We note that this refinement equation was already given in [16] for the standard (nonrational) case. The more general case of a rational operator and of an arbitrary integer dilation factor $m$ (not necessarily a power of two) is considered in [14].

Interestingly, the refinement filter $H_{i}(z)=2\left(c_{i} / c_{i+1}\right)$. $\prod_{k=1}^{N}\left(\left(1+e^{2^{i} \alpha_{k}} z^{-1}\right) / 2\right)$ is now different for each scale; also, it is the same irrespective of the zero vector $\vec{\gamma}$ up to multiplication by a constant. The dual refinement filter $\tilde{H}_{i}(z)$ is given by

$$
\tilde{H}_{i}(z)=\frac{A_{i}(z)}{A_{i+1}\left(z^{2}\right)} H_{i}(z)
$$

and the dual scaling function $\stackrel{\circ}{\varphi}_{i}$ satisfies the relation

$$
\stackrel{\circ}{\varphi}_{i+1}(t)=\sum_{k} \tilde{h}_{i}[k] \stackrel{\circ}{\varphi}_{i}\left(t-2^{i} k\right) .
$$

By substituting (6) into the expression for the autocorrelation sequence at scale $i+1$ given by (4), it is easy to express $A_{i+1}\left(z^{2}\right)$ in terms of $A_{i}(z)$ and $H_{i}(z)$ as

$$
\begin{aligned}
A_{i+1}\left(z^{2}\right)=\frac{1}{2}\left(A_{i}(z) H_{i}(z) H_{i}^{*}\left(z^{-1}\right)\right. \\
\left.+A_{i}(-z) H_{i}(-z) H_{i}^{*}\left(-z^{-1}\right)\right) .
\end{aligned}
$$

\section{B. E-Spline Wavelets}

The inclusion $V_{(i)} \subset V_{(i-1)}$ allows us to uniquely introduce the orthogonal complements $W_{(i)}$ such that

$$
V_{(i)} \oplus W_{(i)}=V_{(i-1)} \text {. }
$$

The residual space $W_{(i)}$ is the orthogonal complement of $V_{(i)}$ in $V_{(i-1)}$. It plays the same role as in the case of semiorthonormal wavelet functions, which are orthonormal across scales, but not necessarily within a given scale. Thus, at a given scale $i+1$, we are looking for a wavelet $\psi_{i+1}$ of the form

$$
\psi_{i+1}(t)=\sum_{k} g_{i}[k] \varphi_{i}\left(t-2^{i} k\right)
$$

that is orthogonal to $V_{i+1}$. In other words, for all $k \in \mathbb{Z}$, we must have

$$
\left\langle\psi_{i+1}(\cdot), \varphi_{i+1}\left(\cdot-2^{i+1} k\right)\right\rangle=0 .
$$

From (9) and (6), and after having expressed the $z$-transform of this orthogonality relation, we obtain

$$
G_{i}(z) H_{i}^{*}\left(z^{-1}\right) A_{i}(z)+G_{i}(-z) H_{i}^{*}\left(-z^{-1}\right) A_{i}(-z)=0 .
$$

In contrast to the classical wavelet theory, all filters now depend on the scale. Hence, the solution $G_{i}(z)$ depends on the scale, too. Its general form is

$$
G_{i}(z)=-z Q_{i}\left(z^{2}\right) H_{i}^{*}\left(-z^{-1}\right) A_{i}(-z)
$$

where $Q_{i}(z)$ is a Laurent polynomial in $z$. We show now how to choose $Q_{i}$ to get a Riesz basis $\left\{\psi_{i}\left(\cdot-2^{i} k\right)\right\}_{k \in \mathbb{Z}}$ of $W_{i}$, while noting that we cannot directly apply Proposition 1 since $\varphi_{i-1}$ and $\psi_{i}$ belong to different functional spaces.

Proposition 2: $\left\{\psi_{i}\left(\cdot-2^{i} k\right)\right\}_{k \in \mathbb{Z}}$ is a Riesz basis of $W_{i}$ if and only if the filter $Q_{i-1}$ is bounded and nonvanishing on the unit circle.

Proof: The autocorrelation filter is

$$
\begin{aligned}
R_{i}\left(e^{2^{i} j \omega}\right) \\
=\sum_{k \in \mathbb{Z}}\left|\hat{\psi}_{i}\left(\omega+\frac{2 \pi}{2^{i}} k\right)\right|^{2} \\
=G_{i-1}\left(e^{2^{i-1} j \omega}\right) A_{i-1}\left(e^{2^{i-1} j \omega}\right) \\
\quad+G_{i-1}\left(e^{j \pi+2^{i-1} j \omega}\right) A_{i-1}\left(e^{j \pi+2^{i-1} j \omega}\right) \\
=A_{i-1}\left(e^{2^{i-1} j \omega}\right) A_{i-1}\left(e^{j \pi+2^{i-1} j \omega}\right)\left|Q_{i-1}\left(e^{2^{i-1} j \omega}\right)\right|^{2} \\
\cdot\left(H_{i-1}\left(e^{2^{i-1} j \omega}\right) A_{i-1}\left(e^{2^{i-1} j \omega}\right)\right. \\
\left.\quad+H_{i-1}\left(e^{j \pi+2^{i-1} j \omega}\right) A_{i-1}\left(e^{j \pi+2^{i-1} j \omega}\right)\right) \\
=A_{i-1}\left(e^{2^{i-1} j \omega}\right) A_{i-1}\left(e^{j \pi+2^{i-1} j \omega}\right) \\
\cdot A_{i}\left(e^{j 2^{i} j \omega}\right)\left|Q_{i-1}\left(e^{2^{i-1} j \omega}\right)\right|^{2} .
\end{aligned}
$$

Thus, for $\left\{\psi_{i}\left(t-2^{i} k\right)\right\}_{k \in \mathbb{Z}}$ to be a Riesz basis in $W_{i}$, there should exist $c_{1}, c_{2}>0$ such that $c_{1} \leq\left|Q_{i-1}\left(e^{2^{i-1} j \omega}\right)\right|^{2}<c_{2}$.

In order to perform a hierarchical decomposition in our new wavelet basis, we would like to apply Mallat's filter bank algorithm. Therefore, we build the filter bank shown in Fig. 6 and impose a perfect reconstruction condition. Following the same construction procedure as before, we obtain the dual wavelet relation

$$
\hat{o}_{i+1}(\omega)=\tilde{G}_{i}\left(e^{2^{i} j \omega}\right) \hat{\hat{\varphi}}_{i}(\omega)
$$

where the dual wavelet filter $\tilde{G}_{i}$ is given by

$$
\tilde{G}_{i}(z)=-z \frac{\tilde{H}_{i}^{*}\left(-z^{-1}\right)}{A_{i}(-z) Q_{i}^{*}\left(z^{-2}\right)} .
$$

We typically use $\left(\tilde{G}_{i}, \tilde{H}_{i}\right)$ on the analysis side and $\left(G_{i}, H_{i}\right)$ on the synthesis side, so that the synthesis wavelet is compactly supported. However, it is possible to interchange these filter 


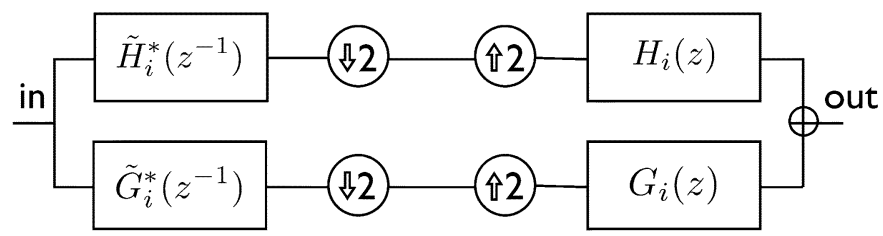

Fig. 6. Two-channel filter bank used for the wavelet transform.

pairs; this corresponds to performing a dual E-spline wavelet transform.

For the orthonormal E-spline wavelet transform, the same filters are used on the analysis and synthesis side. These filters are denoted by $\left(G_{o, i}, H_{o, i}\right)$ and are given by

$$
\begin{aligned}
H_{o, i}(z) & =\sqrt{\frac{A_{i}(z)}{A_{i+1}\left(z^{2}\right)}} H_{i}(z) \\
G_{o, i}(z) & =-z^{-1} H_{o, i}^{*}\left(-z^{-1}\right) .
\end{aligned}
$$

The constructed scaling functions and wavelets at each scale $i$ belong to the space $C^{N-M-2}$. Indeed, they all can be expressed as weighted sums of the shifts of $\varphi_{i-1} \in C^{N-M-2}$ ([14], sec. $3)$. The scaling and the wavelet filters are either finite-impulse response (FIR) (B-spline case) or infinite-impulse response (IIR) with exponential decay (dual or orthogonal case); thus, the basis functions are either compactly supported, or decay exponentially.

\section{Properties of E-Splines AND E-Spline WaVElets}

\section{A. Reproduction of Exponential Polynomials}

The following proposition generalizes the polynomial-reproduction property of the classical wavelet theory:

Property 1: Suppose that $\vec{\alpha}$ contains a root $\alpha_{0}$ of multiplicity $m$. Then, for $n=0, \ldots, m-1$ the exponential monomial $t^{n} e^{\alpha_{0} t}$ has the B-spline representation

$$
t^{n} e^{\alpha_{0} t}=\sum_{k \in \mathbb{Z}} p_{\vec{\alpha}, i, n}[k] \beta_{\vec{\alpha}, \vec{\gamma}, 2^{i}}\left(t-2^{i} k\right)
$$

where $p_{\vec{\alpha}, i, n}[k]$ are suitable coefficients.

For the arbitrary parameter vector $\vec{\alpha}$ consisting of $N_{d}$ distinct roots of multiplicity $m_{k}, k=1, \ldots, N_{d}$, the direct corollary is that the exponential polynomials

$$
p_{\vec{\alpha}}(t)=\sum_{k=1}^{N_{d}} \sum_{n=0}^{m_{k}-1} c_{k} t^{n} e^{\alpha_{k} t}
$$

that constitute the null space $\mathcal{N}_{\vec{\alpha}}$ of the operator L can be reproduced with B-splines. The result follows from Proposition 2 in [13].

\section{B. Vanishing Exponential Moments}

In conventional wavelet theory, the vanishing-moment property of the wavelet is closely related to the ability of the scaling function to reproduce polynomials. This can be generalized as well to our case of rational operators.
Property 2: For each scale $i \in \mathbb{Z}$, shift $t_{0} \in \mathbb{R}$, and degree $n=$ $0, \ldots, m_{k}-1$, the analysis wavelet satisfies

$$
\int_{-\infty}^{\infty} t^{n} e^{\alpha_{k} t} \psi_{i}^{*}\left(t-t_{0}\right) \mathrm{d} t=0
$$

where $m_{k}$ is the multiplicity of $\alpha_{k}$. In other words, the analysis wavelet $\stackrel{\circ}{i}_{i}^{*}$ has $N$ vanishing exponential moments that correspond to the basis functions of the null space of L. Equivalently, for each $p_{\vec{\alpha}}(t) \in \mathcal{N}_{\vec{\alpha}}$, we have

$$
\int_{-\infty}^{\infty} p_{\vec{\alpha}}(t) \stackrel{\circ}{\psi_{i}^{*}}\left(t-t_{0}\right) \mathrm{d} t=0 .
$$

This proposition becomes obvious if we remember that $W_{(i)}$ is an orthogonal complement of $V_{(i)}$; thus, as long as the null space of $\mathrm{L}$ can be reconstructed with the basis functions of $V_{(i)}, \hat{\psi}_{i}$ is orthogonal to it. In addition, the null space is shift-invariant [14]; i.e., $p_{\vec{\alpha}}\left(t-t_{0}\right) \in \mathcal{N}_{\vec{\alpha}}$, which completes the proof.

\section{Operator-Like Wavelets}

The following theorem is a key result of the present work. It states that our new wavelets behave qualitatively like the differential operator from which the multiresolution analysis is derived.

Theorem 2: Let $\left\{\psi_{i, k}\right\}_{i, k \in \mathbb{Z}}$ be an E-spline wavelet basis of $L_{2}$. Then, there exists a sequence $\left\{\phi_{i}\right\}_{i \in \mathbb{Z}}$ of E-spline scaling functions of order $2 N$ such that $\left\langle f, \psi_{i}\left(\cdot-t_{0}\right)\right\rangle=\mathrm{L}\left\{f * \phi_{i}\right\}\left(t_{0}\right)$; in addition, $\left\{\phi_{i}\right\}_{i \in \mathbb{Z}}$ generates a multiresolution analysis of $L_{2}$. The wavelet coefficients of $f$ are therefore the samples of the smoothed versions of $L_{\vec{\alpha}, \vec{\gamma}} f$.

Proof: Consider the wavelet coefficient $\left\langle f, \stackrel{\circ}{\psi}_{i}\left(\cdot-t_{0}\right)\right\rangle=$ $\left.\int_{\hat{o}} L(j \omega) \hat{f}(\omega) \hat{\circ}_{i}^{*}(\omega) / L(j \omega)\right) e^{j \omega t_{0}} \mathrm{~d} \omega$. We now define $\hat{\phi}_{i}(\omega)=$ $\psi_{i}^{*}(\omega) / L(j \omega)$ and study the behavior of this function. Combining (7) and (10), we get

$$
\begin{aligned}
& \hat{\phi}_{i}(\omega)=\frac{\tilde{G}_{i-1}^{*}\left(e^{-j 2^{i-1} \omega}\right)}{L(j \omega)} \hat{o}_{i-1}^{*}(\omega) \\
& =-e^{-j 2^{i-1} \omega} \frac{H_{i-1}\left(-e^{j 2^{i-1} \omega}\right)}{L(j \omega) A_{i}\left(e^{j 2^{i} \omega}\right) Q_{i-1}\left(e^{j 2^{i} \omega}\right)} \hat{\grave{\varphi}}_{i-1}^{*}(\omega) .
\end{aligned}
$$

In order to further simplify this expression, we notice that $\hat{\varphi}_{i}(\omega)=\left(H_{i}\left(-e^{j 2^{i} \omega}\right) / L(j \omega)\right)$. Hence, taking (5) into account, we have

$$
\hat{\phi}_{i}(\omega)=-\left.e^{-j 2^{i-1} \omega} \frac{A_{i-1}\left(e^{j 2^{i-1} \omega}\right)}{A_{i}\left(e^{j 2^{i} \omega}\right) Q_{i-1}\left(e^{j 2^{i} \omega}\right)} \hat{\hat{\varphi}}_{i-1}(\omega)\right|^{2} .
$$

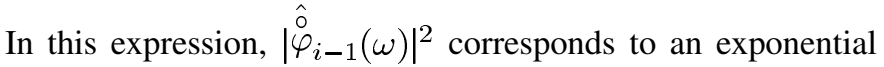
B-spline with augmented parameters $\left(\vec{\alpha}:-\vec{\alpha}^{*}\right),\left(\vec{\gamma}:-\vec{\gamma}^{*}\right)$. The discrete filters $A_{i-1}, A_{i}$, and $Q_{i-1}$, are bounded and do not vanish on the unit circle. According to Proposition $1,\left\{\phi_{i}(\cdot-\right.$ $\left.\left.2^{i-1} k\right)\right\}_{k \in \mathbb{Z}}$ is a Riesz basis of the space generated by $\stackrel{0}{\varphi}_{i-1} *$ $\stackrel{\circ}{\varphi}_{i-1}^{T}$, which implies that the $\phi_{i}$ 's generate a multiresolution analysis of $L_{2}$. 
When $\gamma_{k} \neq 0$ for all $k$ and $\alpha_{l} \neq 2^{-i} \cdot 2 \pi k j$ for $1 \leq l \leq$ $N, k \neq 0$, then the filters $\phi_{i}$ are low pass. The sufficient condition is that $\varphi_{i}$ is a valid E-spline scaling function with $\alpha_{l}=0$ for at least one $l$. The validity statement then implies all other requirements. This is exactly the case for the examples in Section VI.

\section{IMPLEMENTATION AND EXAMPLES}

In this section, we describe the filter bank implementation for the wavelet decomposition and reconstruction based on E-splines. We also discuss possible algorithms for the calculation of the autocorrelation filter. Finally, we show examples of exponential B-splines, illustrating the concepts of this paper.

\section{A. Filter Bank Implementation}

In practice, it is more efficient to work with discrete sequences and filter banks, rather than with continuous-time signals and projections. In the conventional wavelet theory, this idea leads to Mallat's fast filter bank algorithm [1]. It is easy to see that this algorithm can be applied to the E-spline-wavelet case as well; however, the filters $\left(G_{i}, \tilde{G}_{i}\right)$ and $\left(H_{i}, \tilde{H}_{i}\right)$ must be precalculated for each iteration. IIR filters can be implemented recursively (as in [9]) or approximated with FIR filters of sufficient length. A simple alternative is to evaluate these in the Fourier domain using the fast Fourier transform (FFT) algorithm [24].

The algorithm first interpolates the given samples $f[k]$ with the exponential B-splines at the initial scale $i=0$

$$
f_{0}(t)=\sum_{k \in \mathbb{Z}} c[k] \varphi_{0}(t-k)
$$

where $c[k]=(p * f)[k]$, and where $P(z)$ is the interpolation prefilter given by

$$
P(z)=\frac{1}{\left(\sum_{k} \varphi_{0}(k) z^{-k}\right)} .
$$

The $c[k]$ 's are used to initialize the hierarchical decomposition.

In the unlikely event where $P(z)$ is not stable, which is also sometimes the case with conventional splines, we propose to replace the filter by a generalized quasi-interpolant [25] that is specifically designed to reproduce the exponential polynomials.

The (quasi-)interpolation model (11) implies that $f_{0} \in V_{0}$. Thus, the $c[k]$ 's given by the initialization procedure are also the coefficients of the projection of $f_{0}$ into $V_{0}$. Indeed

$$
\left\langle f_{0}, \stackrel{\circ}{\varphi}_{0}(\cdot-k)\right\rangle=\sum_{k_{0} \in \mathbb{Z}} c\left[k_{0}\right]\left\langle\varphi_{0}\left(\cdot-k_{0}\right), \stackrel{\circ}{\varphi}_{0}(\cdot-k)\right\rangle=c[k]
$$

because of the biorthogonality of $\varphi_{0}$ and $\stackrel{\circ}{\varphi}_{0}$.

For each iteration, we need to know the autocorrelation filter $A_{i}(z)$ at the current scale $i$. Then, the wavelet filters $G_{i}(z), \tilde{G}_{i}(z), G_{o, i}$, and the scaling filters $H_{i}(z), \tilde{H}_{i}(z), H_{o, i}(z)$, can be computed using the explicit expressions given in Section IV. Equation (8) allows us to find $A_{i+1}(z)$ from $A_{i}(z)$. For $A_{0}(z)$, we recall that the autocorrelation function of the exponential B-spline corresponds to the scaled symmetrical B-spline with parameters $\left(\vec{\alpha},-\vec{\alpha}^{*}\right),\left(\vec{\gamma},-\vec{\gamma}^{*}\right)[13]$.
We see that, both for the autocorrelation filter and for the interpolation prefilter, we need to compute the exponential B-spline samples in the time domain. This can be done by evaluating the samples of the Green's function and by applying finite-difference operators to them.

In the first order case, the procedure is especially simple because $A_{i}(z)=1$. In that case, $H_{i}(z)=\tilde{H}_{i}(z)=H_{\alpha, i}(z)=$ $\left(1 / \sqrt{1+\left|e^{2^{i+1} \alpha}\right|}\right) \cdot\left(1+e^{2^{i} \alpha} z^{-1}\right)$ and $G_{i}(z)=\tilde{G}_{i}(z)=$ $\left(1 / \sqrt{1+\left|e^{2 i+1} \alpha\right|}\right)\left(e^{2^{i} \alpha^{*}}-z^{-1}\right)$, where we observe the simple dependence on the scale. Interestingly, the filters tend to those of the Haar system (sum and difference) as the scale gets finer $(i \rightarrow-\infty)$.

\section{B. Computation of the Green's Function Samples}

For $\operatorname{Re}\left\{\alpha_{k}\right\}>0$, the Green's function grows exponentially. To deal with this issue, we use anticausal Green's functions for all $k$ such that $\operatorname{Re}\left\{\alpha_{k}\right\}>0$, tuning the sign in the partial fraction decomposition. Having decomposed $\hat{\rho}(\omega)$ into partial fractions

$$
\hat{\rho}(\omega)=\sum_{n=1}^{N_{d}} \sum_{l=1}^{m_{n}} \frac{c_{n, l}}{\left(j \omega-\alpha_{n}\right)^{l}}
$$

we express the components with $\operatorname{Re}\left\{\alpha_{k}\right\}>0$ as

$$
\frac{c_{n, l}}{\left(j \omega-\alpha_{n}\right)^{l}}=\frac{(-1)^{l} c_{n, l}}{\left(-j \omega-\left(-\alpha_{n}\right)\right)^{l}} \text {. }
$$

In the time domain, the latter corresponds to anticausal Green's function with parameter $\alpha_{n}$.

We further notice that, to calculate $N$ samples of the B-spline, we need $2 N+1$ values of the Green's function; thus, $\rho(t)$ should be calculated for $t_{k}=-N, \ldots, N$.

\section{Computation of the Exponential B-Spline Samples and Filters}

We apply $N$ finite-difference operators $\Delta_{\left(\alpha_{1}\right), 2^{i}}, \ldots$, $\Delta_{\left(\alpha_{N}\right), 2^{i}}$ to the Green's function samples and obtain $N$ samples of the exponential B-spline. The value $c_{i}=\sqrt{a_{i}[0]}$ is equal to the norm of the B-spline and is used for normalization. The autocorrelation filter is obtained by taking the value of the trigonometric polynomial

$$
A_{i}\left(e^{2^{i} j \omega}\right)=\sum_{k=-N}^{N} a_{i}[k] e^{2^{i} j \omega k} .
$$

Since the samples of the exponential B-spline are known, the interpolation prefilter can be computed from (12).

\section{Examples}

For our first example, we choose $\vec{\alpha}=(0,-1)$ and $\vec{\gamma}$ empty. We show the basic and dual scaling functions and the wavelets in Fig. 7(a)-(d). It can be seen that these functions are nonsymmetric, piecewise exponential, and yet continuous because $N-M=2$. The two synthesis functions $\varphi_{0}$ and $\psi_{1}$ are compactly supported, while their dual counterparts $\stackrel{\circ}{\varphi}_{0}$ and $\stackrel{\circ}{\psi}_{1}$ are exponentially decaying. 


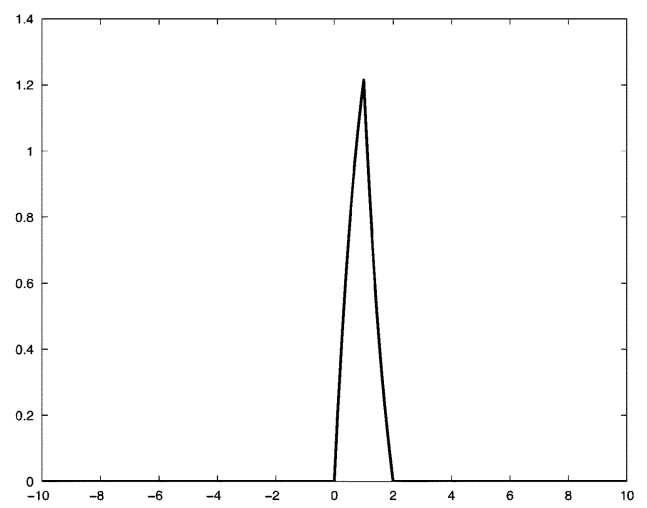

(a)

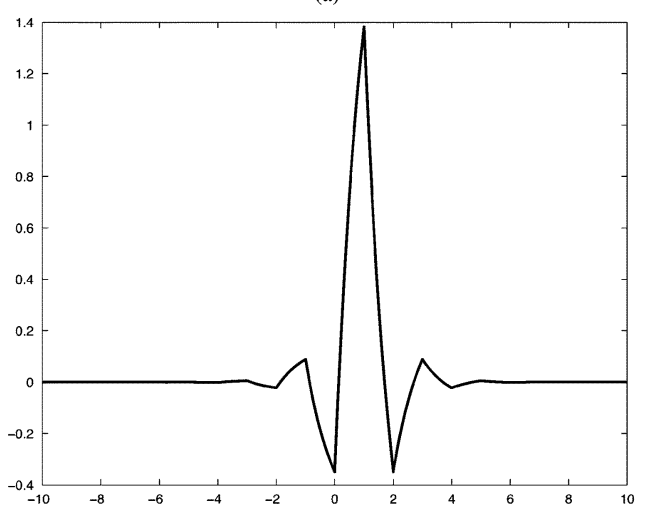

(b)

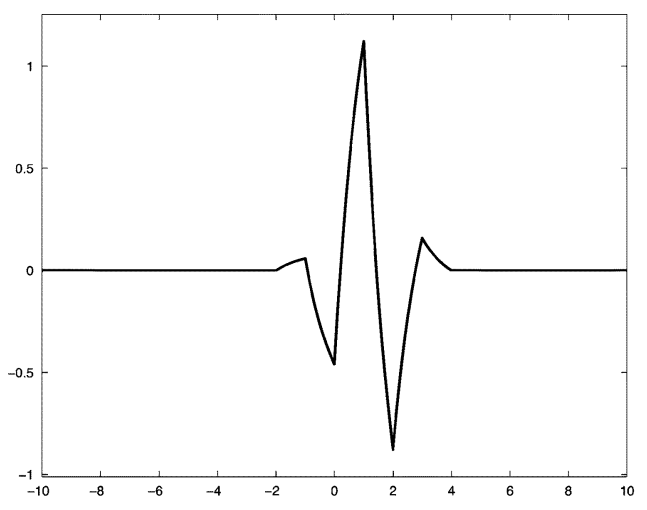

(c)

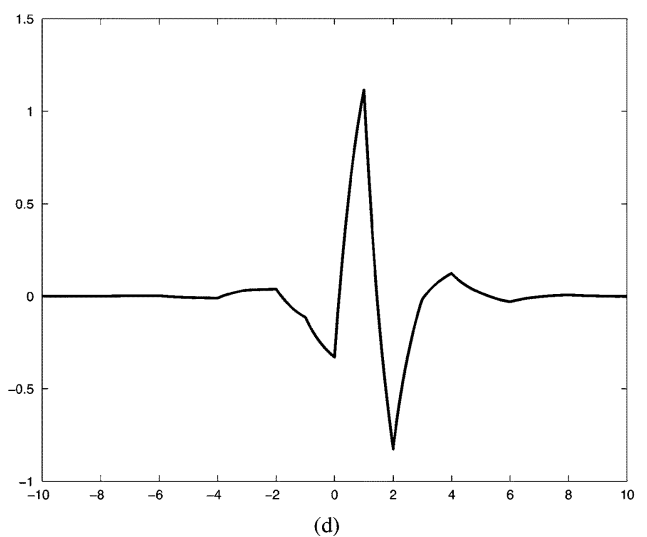

(d)

Fig. 7. Example 1: Basic and dual scaling functions and wavelets; $\vec{\alpha}=(0,-1), \vec{\gamma}$ is empty. (a) Scaling function $\varphi_{0}(t)$. (b) Dual scaling function $\dot{\varphi}_{0}(t)$. (c) Synthesis wavelet $\psi_{1}(t)$. (d) Analysis wavelet $\dot{ }_{1}(t)$.

The spectrum of the analysis wavelet $\stackrel{\circ}{\psi}$ is displayed in Fig. 8(a). The frequency response of the corresponding op-

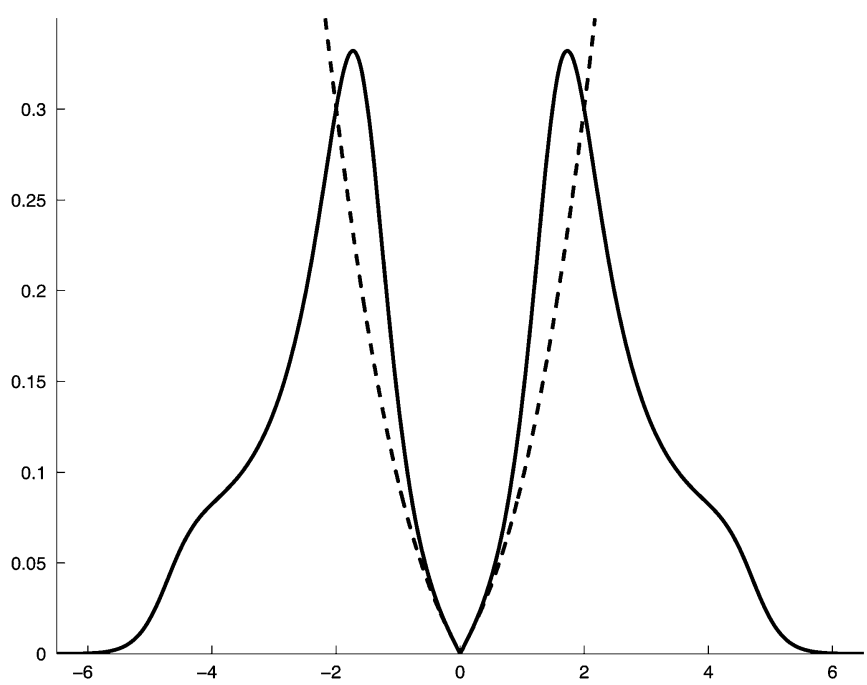

(a)

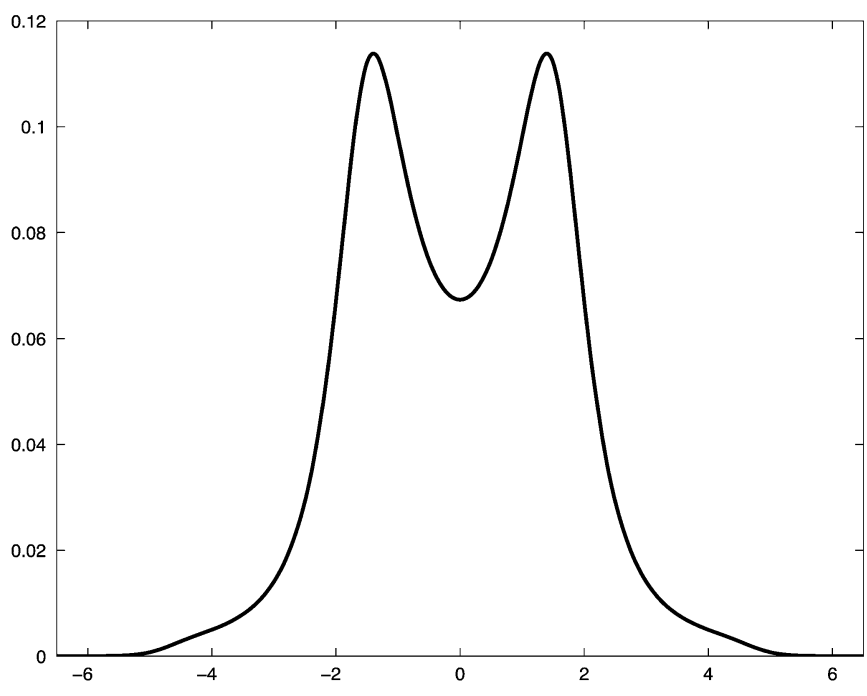

(b)

Fig. 8. Example 1: Spectral behavior of the analysis wavelet and of the smoothing kernel; $\vec{\alpha}=(0,-1), \vec{\gamma}$ is empty. (a) Spectrum of the analysis wavelet $\left|\hat{\mathrm{\psi}}_{1}(\omega)\right|$ and $|L(j \omega)|$. (b) Spectrum $\left|\hat{\phi}_{1}(\omega)\right|$ of the smoothing kernel.

erator $\mathrm{L}=\mathrm{D}+\mathrm{D}^{2}$ is overlaid with a dashed line. It can be seen that the two plots are well matched around the origin (at the location of the pole $j \omega=\alpha_{1}=0$ ), which illustrates the differential-operator property (see Theorem 2 ). We show the spectrum of the smoothing kernel $\left|\hat{\phi}_{1}(\omega)\right|=\left|\hat{\psi}_{1}^{*}(\omega) / L(j \omega)\right|$ in Fig. 8(b); it is clearly low pass and decays rapidly for high frequencies.

With our second example, we illustrate the spectral behavior of the wavelets and of the scaling functions. For this purpose, we choose the parameters $\vec{\alpha}=$ $(-(5 \pi / 8) j,-(5 \pi / 8) j,(5 \pi / 8) j,(5 \pi / 8) j, 0,0)$ and $\vec{\gamma}=$ $(-5 j, 5 j)$ to be pure imaginary and to have Hermitian symmetry, which ensures that the time-domain functions are real. In Fig. 9, we show the scaling function $\varphi_{0}$ and the wavelets $\psi_{1}$ and $\psi_{2}$ in the time domain; these have a strong oscillatory character, with a larger number of lobes as the scale gets coarser. It is clearly apparent that $\psi_{2}$ is not a dilate of $\psi_{1}$. Figs. 7 and 


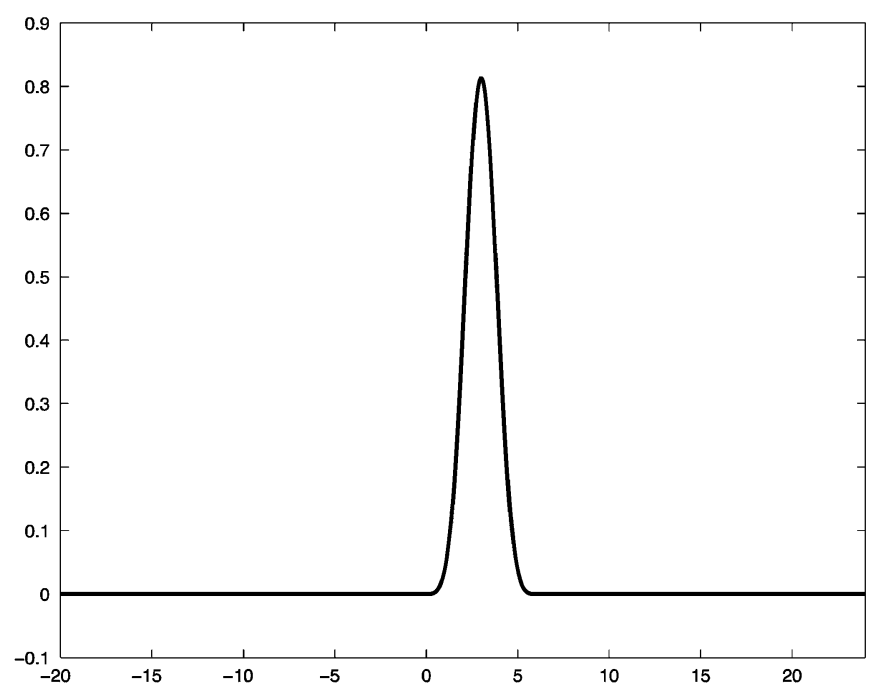

(a)

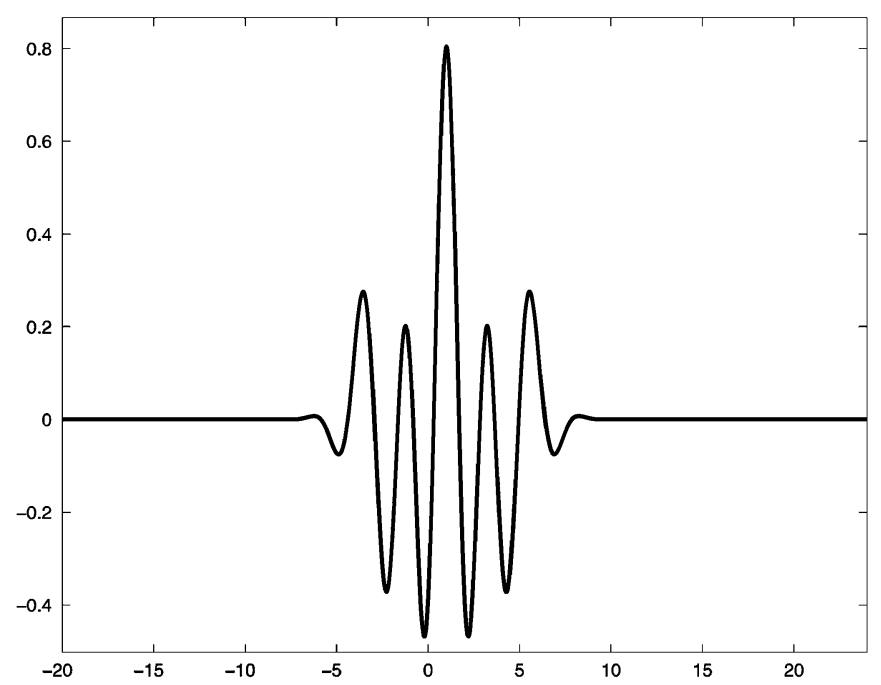

(b)

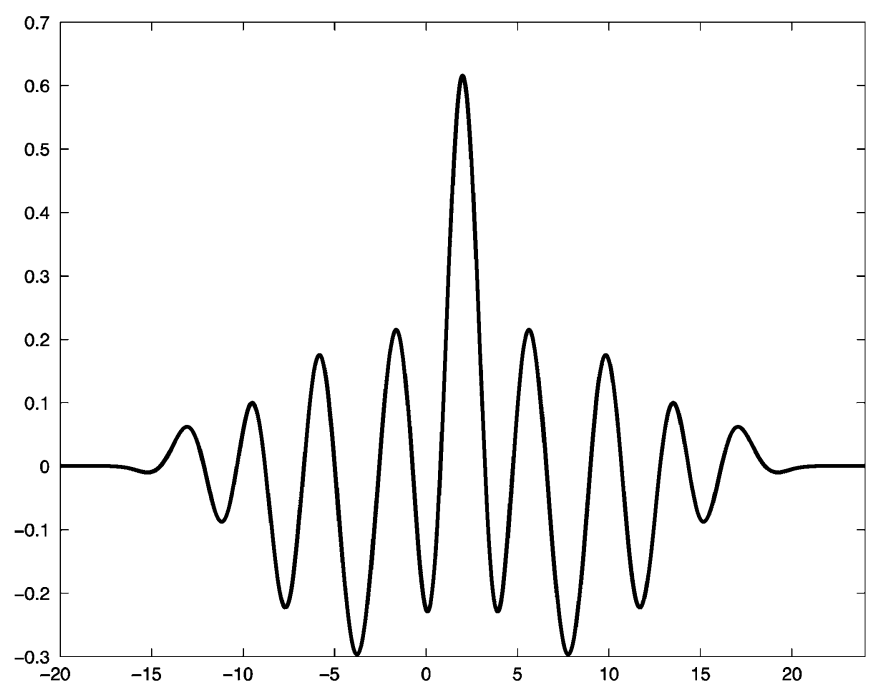

(c)

Fig. 9. Example 2: Scaling function at the scale $i=0$ and the wavelets at the scales $i=1, i=2 ; \vec{\alpha}=(-(5 \pi / 8) j,-(5 \pi / 8) j$, $(5 \pi / 8) j,(5 \pi / 8) j, 0,0), \vec{\gamma}=(-5 j, 5 j)$. (a) Scaling function $\varphi_{0}, i=0$. (b) Wavelet $\psi_{1}, i=1$. (c) Wavelet $\psi_{2}, i=2$.

9 illustrate the diversity of shapes that can be obtained with E-spline scaling functions and wavelets.

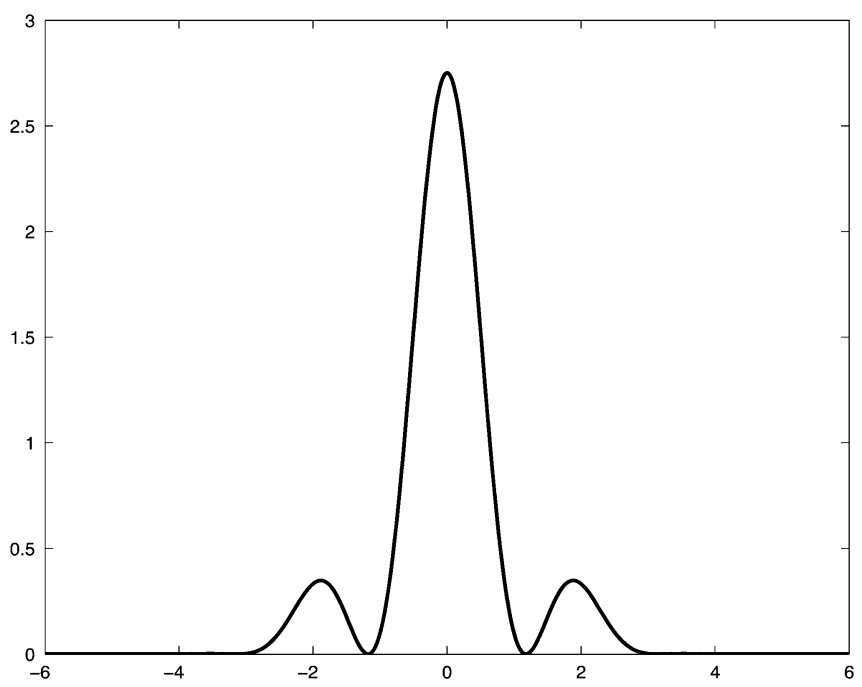

(a)

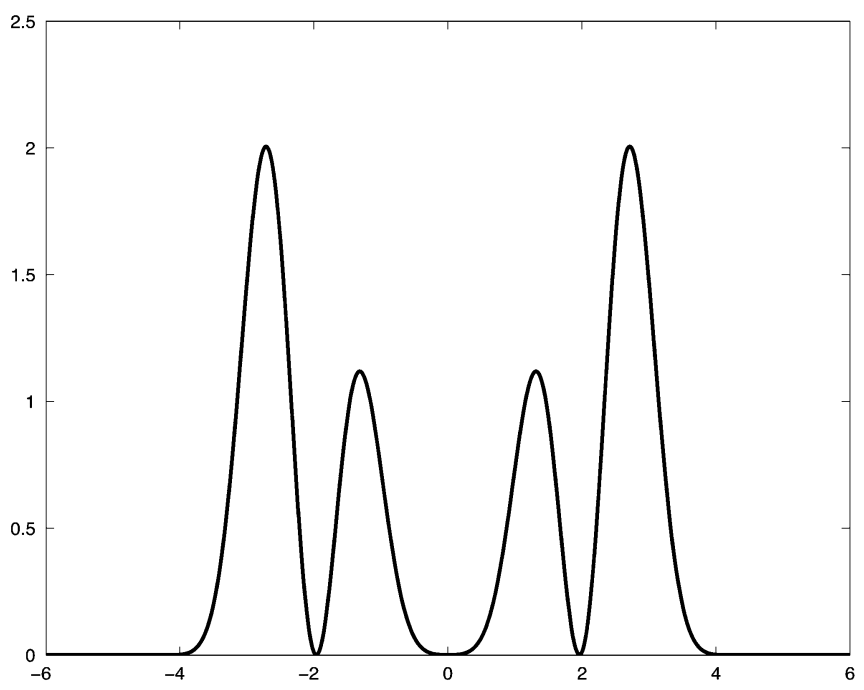

(b)

Fig. 10. Example 2: Spectrum of the scaling function and the spectrum of the wavelet; $\vec{\alpha}=(-(5 \pi / 8) j,-(5 \pi / 8) j,(5 \pi / 8) j,(5 \pi / 8) j, 0,0), \vec{\gamma}=$ $(-5 j, 5 j)$. (a) Scaling function spectrum $\left|\hat{\varphi}_{1}(\omega)\right|, i=1$. (b) Wavelet spectrum $\left|\hat{\psi}_{1}(\omega)\right|, i=1$.

The plots in Fig. 10 show the effect of the poles $\vec{\alpha}$ and of the zeros $\vec{\gamma}$ on the frequency response. In Fig. 10(a), the pole $\alpha_{1}=-(5 \pi / 8) j$ produces a peak of the scaling-function spectrum near $\omega=-(5 \pi / 8) j$ and makes it vanish with periodicity $\pi$. There is also a peak at the origin because of the pole at $\omega=0$. The zero $\gamma_{1}=-5 j$ makes the frequency response vanish at $\omega=-5$. In contrast, the wavelet spectrum [Fig. 10(b)] vanishes at $j \omega=\alpha_{l}$, as a result of the vanishing exponential moment and of the linear-differential-operator properties of E-spline wavelets.

\section{CONCLUSION}

In this paper, we have introduced a new procedure for constructing wavelet-like bases from linear differential operators. Similar to analog filters, the wavelet spaces are characterized by the poles and zeros of the underlying operator. The wavelets 
come in three different flavors: basic (B-spline), dual, and orthonormal. We have studied the approximation-order properties of the multiresolution analysis. These wavelet bases possess powerful properties, including the ability to kill exponential polynomials, which generalizes the notion of the vanishing moments found in the conventional wavelet theory. More importantly, they essentially behave as multiscale versions of the underlying operator. We recover the polynomial B-spline case by choosing the parameter $\vec{\alpha}=(0, \ldots, 0)$, where the corresponding operator is the $N$ th derivative.

The constructed wavelets are not dilates of a single function anymore; however, they still can be implemented using a nonstationary version of Mallat's fast filter bank algorithm.

The proposed framework should be of interest for signal processing applications. It might be well suited to signals that are not predominantly low pass but that have substantial energy concentrations in some frequency bands. In particular, it offers the possibility of adapting the model for a given class of signals, by selecting roots that fit the natural resonances of the data.

\section{APPENDIX}

\section{A. Proof of Theorem 1}

First, we outline some basic notations used in [13] to be able to perform the estimations. The spectrum of the first-order E-spline $\left|\hat{\beta}_{\sigma+j \omega_{0}}(\omega)\right|$ achieves its maximum $M_{\sigma+j \omega_{0}}$ at $\omega=\omega_{0}$. For the $N$ th-order case, we denote $M_{\vec{\alpha}}=\prod_{l=1}^{N} M_{\alpha_{l}}$.

By referring to the general approximation results of Blu et al. [26], we consider the limit

$$
\begin{aligned}
\lim _{T \rightarrow 0} \frac{\left\|f-P_{T} f\right\|_{L_{2}}^{2}}{T^{2(N-M)}}=\lim _{T \rightarrow 0} \int_{-\infty}^{\infty} \sum_{k \neq 0}|\hat{f}(\omega)|^{2} \\
\times \frac{\left|\hat{\beta}_{T \vec{\alpha}, T \vec{\gamma}}(\omega T+2 \pi k)\right|^{2} / T^{2 N}}{\sum_{k}\left|\hat{\beta}_{T \vec{\alpha}, T \vec{\gamma}}(\omega T+2 \pi k)\right|^{2} / T^{2 M}} \mathrm{~d} \omega .
\end{aligned}
$$

We denote $\Psi_{1}(k, T, \omega)=\left(\hat{\beta}_{T \vec{\alpha}, T \vec{\gamma}}(\omega T+2 \pi k)\right) / T^{N}$ and $\Psi_{2}(k, T, \omega)=\left(1 / T^{M}\right) \sqrt{\sum_{k}\left|\hat{\beta}_{T \vec{\alpha}, T \vec{\gamma}}(\omega T+2 \pi k)\right|^{2}}$.

To evaluate (13), we would like to exchange the $\lim _{T \rightarrow 0}$ and $\int \sum$ signs. To be able to apply Lebesgue's theorem, we must first prove that $\left|\left(\Psi_{1}(k, T, \omega) / \Psi_{2}(k, T, \omega)\right) \hat{f}(\omega)\right|^{2}$ is bounded by a summable and integrable function that does not depend on $T$. As we are interested in small values of $T$ only, we assume that

$$
T<\min \left\{\frac{\pi}{\max _{l}\left|\gamma_{l}\right|+\max _{l}\left|\alpha_{l}\right|}, \frac{\pi}{3 \max _{l}\left|\alpha_{l}\right|}\right\} .
$$

Then, for $\Psi_{1}(k, T, \omega)$, we have that

$$
\begin{array}{r}
\Psi_{1}(k, T, \omega)=\prod_{l=1}^{M} \frac{j \omega T-\gamma_{l} T+2 \pi k}{T\left(j \omega T-\alpha_{l} T+2 \pi k\right)}\left(1-e^{T \alpha_{l}-T j \omega+2 \pi k}\right) \\
\cdot \prod_{l=M+1}^{N} \frac{1-e^{T \alpha_{l}-T j \omega+2 \pi k}}{T\left(j \omega T-\alpha_{l} T+2 \pi k\right)}
\end{array}
$$

We perform the partial fraction decomposition

$$
\begin{aligned}
& \frac{j \omega T-\gamma_{l} T+2 \pi k}{\left(j \omega T-\alpha_{l} T\right)\left(j \omega T-\alpha_{l} T+2 \pi k\right)} \\
& =\frac{\left(\alpha_{l}-\gamma_{l}\right) T / 2 \pi k+1}{\left(j \omega T-\alpha_{l} T+2 \pi k\right)}+\frac{-\left(\alpha_{l}-\gamma_{l}\right) T / 2 \pi k}{\left(j \omega T-\alpha_{l} T\right)} .
\end{aligned}
$$

From ([13], Appendix A), we know that $\left|1-e^{T \alpha_{l}-T j \omega+2 \pi k}\right| /\left|\left(j \omega T-\alpha_{l} T\right)\left(j \omega T-\alpha_{l} T+2 \pi k\right)\right| \leq$ $\left(M_{\alpha_{l}} \pi|k|\right)$. Thus, taking into account (14), we get the following estimation for $\Psi_{1}$ :

$$
\begin{aligned}
& \left|\Psi_{1}(k, T, \omega)\right| \\
& \leq \frac{1}{T^{N}}\left|L_{T \vec{\alpha}}(j \omega T)\right| \prod_{l=1}^{M} M_{T \alpha_{l}}\left(1+\frac{\left|\alpha_{l}-\gamma_{l}\right| T}{\pi|k|}\right) \\
& \quad \cdot \prod_{l=M+1}^{N} \frac{M_{\alpha_{l}}}{\pi|k|} \leq\left|L_{\vec{\alpha}}(j \omega)\right| \frac{2^{M} M_{\vec{\alpha}}}{(\pi|k|)^{N-M}} .
\end{aligned}
$$

We now bound $\Psi_{2}$ from below. Using a technique similar to the one in [13] for the lower Riesz-bound estimation, we obtain

$$
\frac{\Psi_{2}(k, T, \omega)}{\left|\prod_{l=1}^{M}\left(j \omega-\gamma_{l}\right)\right|} \geq \inf _{\omega \in\left[-\frac{\pi}{T}, \frac{\pi}{T}\right]}\left|\hat{\beta}_{T \vec{\alpha}}(\omega T)\right| \geq \frac{M_{T \vec{\alpha}}}{\pi^{N}} .
$$

Consequently, for $\left|\left(\Psi_{1} / \Psi_{2}\right) \hat{f}\right|^{2}$ we get the bound

$$
\left|\frac{\Psi_{1}(k, T, \omega)}{\Psi_{2}(k, T, \omega)} \hat{f}(\omega)\right|^{2} \leq(2 \pi)^{2 M} \frac{\left|L_{\vec{\alpha}, \vec{\gamma}}(j \omega) \hat{f}(\omega)\right|^{2}}{|k|^{2(N-M)}}
$$

which is summable over $k$ and integrable over $\omega$ under the assumptions of the theorem.

We thus exchange the limit with the integration and summation and calculate

$$
\begin{aligned}
\lim _{T \rightarrow 0}\left|\frac{\Psi_{1}(k, T, \omega)}{\Psi_{2}(k, T, \omega)} \hat{f}(\omega)\right|^{2} \\
=\left|\frac{\hat{f}(\omega) \prod_{l=1}^{N}\left(j \omega-\alpha_{l}\right)}{(2 \pi k)^{N-M} \prod_{l=1}^{M}\left(j \omega-\gamma_{l}\right)}\right|^{2} \\
=\frac{\left|L_{\vec{\alpha}, \vec{\gamma}}(j \omega) \hat{f}(\omega)\right|^{2}}{(2 \pi k)^{2(N-M)}},
\end{aligned}
$$

which yields the desired result

$$
\lim _{T \rightarrow 0} \frac{\left\|f-P_{T} f\right\|_{L_{2}}^{2}}{T^{2(N-M)}}=C_{N, M}^{2}\left\|L_{\vec{\alpha}, \vec{\gamma}} f\right\|_{L_{2}}^{2} .
$$

\section{ACKNOWLEDGMENT}

The authors would like to thank the anonymous reviewer for pointing out to them two references that are quite relevant to this research [19], [20]. They also would like to thank T. Blu, who provided them with mathematical advice.

\section{REFERENCES}

[1] S. Mallat, A Wavelet Tour of Signal Processing. San Diego, CA: Academic, 1998.

[2] G. Strang and T. Nguyen, Wavelets and Filter Banks. Wellesley, MA: Wellesley-Cambridge, 1996. 
[3] M. Vetterli and J. Kovačević, Wavelets and Subband Coding. Englewood Cliffs, NJ: Prentice-Hall, 1995.

[4] I. Daubechies, "Orthogonal bases of compactly supported wavelets," Commun. Pure Appl. Math., vol. 41, pp. 909-996, 1988.

[5] — " "Orthonormal bases of compactly supported wavelets II. Variations on a theme," SIAM J. Math. Anal., vol. 24, no. 2, pp. 499-519, Mar. 1993.

[6] A. Cohen, I. Daubechies, and J. C. Feauveau, "Bi-orthogonal bases of compactly supported wavelets," Commun. Pure Appl. Math., vol. 45, pp. 485-560, 1992.

[7] G. Battle, "A block spin construction of ondelettes. Part I: Lemarié functions," Commun. Math. Phys., vol. 110, pp. 601-615, 1987.

[8] P.-G. Lemarié, "Ondelettes à localization exponentielle," J. Math. Pures. Appl., vol. 67, no. 3, pp. 227-236, 1988.

[9] M. Unser, A. Aldroubi, and M. Eden, "A family of polynomial spline wavelet transforms," Signal Process., vol. 30, no. 2, pp. 141-162, Jan. 1993.

[10] — "On the asymptotic convergence of B-spline wavelets to Gabor functions," IEEE Trans. Inf. Theory, vol. 38, no. 2, pp. 864-872, Mar. 1992.

[11] M. Unser and T. Blu, "Wavelet theory demystified," IEEE Trans. Signal Process., vol. 51, no. 2, pp. 470-483, Feb. 2003.

[12] L. L. Schumaker, Spline Functions: Basic Theory. New York: Wiley, 1981.

[13] M. Unser and T. Blu, "Cardinal exponential splines: Part I-Theory and filtering algorithms," IEEE Trans. Signal Process., vol. 53, no. 4, pp. 1425-1438, Apr. 2005.

[14] M. Unser, "Cardinal exponential splines: Part II-Think analog, act digital," IEEE Trans. Signal Process., vol. 53, no. 4, pp. 1439-1449, Apr. 2005.

[15] J. Stöckler, "Non-stationary wavelets," Multivariate Approximation: From CAGD to Wavelets, pp. 307-320, 1993.

[16] C. de Boor, R. A. DeVore, and A. Ron, "On the construction of multivariate (pre)wavelets," Constr. Approx., vol. 2, no. 3, pp. 123-166, 1993.

[17] C. K. Chui, J. Stöckler, and J. D. Ward, "Analytic wavelets generated by radial functions," Adv. Comput. Math., vol. 5, pp. 95-123, 1996.

[18] A. Cohen and N. Dyn, "Nonstationary subdivision schemes and multiresolution analysis," SIAM J. Math. Anal., vol. 27, no. 6, pp. 1745-1769, Nov. 1996.

[19] T. Lyche and L. L. Schumaker, "L-spline wavelets," in Wavelets: Theory, Algorithms, and Applications, C. K. Chui, L. Montefusco, and L. Puccio, Eds. New York: Academic, 1994, pp. 197-212

[20] S. Dahlke, W. Dahmen, E. Schmitt, and I. Weinreich, "Multiresolution analysis and wavelets on $S^{2}$ and $S^{3}$," Numer. Funct. Anal. Optim., vol. 16, no. 1\&2, pp. 19-41, 1995.

[21] N. Dyn, D. Levin, and A. Luzzatto, "Exponentials reproducing subdivision schemes," Found. Comput. Math., vol. 3, pp. 187-206, 2003.

[22] N. Dyn and A. Ron, "Local approximation by certain spaces of exponential polynomials, approximation order of exponential box splines, and related interpolation problems," Trans. Amer. Math. Soc., vol. 319, pp. 381-403, 1990.

[23] M. Unser and T. Blu, "Mathematical properties of the JPEG2000 wavelet filters," IEEE Trans. Image Process., vol. 12, no. 9, pp. 1080-1090, Sep. 2003
[24] T. Blu and M. Unser, "The fractional spline wavelet transform: Definition and implementation," in Proc. 25th IEEE Int. Conf. Acoustics, Speech, Signal Processing (ICASSP), vol. I, Istanbul, Turkey, Jun. 5-9, 2000, pp. 512-515.

[25] C. de Boor, "Quasiinterpolation and approximation power of multivariate splines," in Computation of Curves and Surfaces. Dordrecht, Germany: Kluwer, 1990, pp. 313-345.

[26] T. Blu and M. Unser, "Quantitative Fourier analysis of approximation techniques: Part I-Interpolators and projectors," IEEE Trans. Signal Process., vol. 47, no. 10, pp. 2783-2795, Oct. 1999.

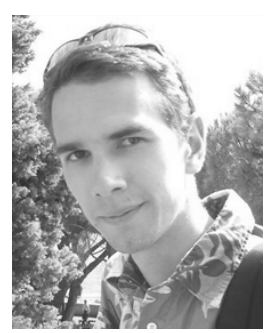

Ildar Khalidov was born in St.-Petersburg, Russia, in 1982. He received the Master's degree in mathematics and computer science from St.-Petersburg State University, St.-Petersburg, Russia, in 2003.

$\mathrm{He}$ is now with the Biomedical Imaging Group, Swiss Federal Institute of Technology Lausanne (EPFL), Switzerland. His current research interests include splines, wavelets, and biomedical signal processing, and imaging applications, such as MRI/MRSI and ultrasound imaging.

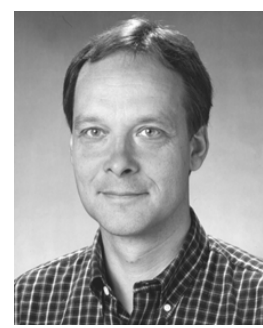

Michael Unser (M'89-SM'94-F'99) received the M.S. (summa cum laude) and Ph.D. degrees in electrical engineering from the Ecole Polytechnique Fédérale de Lausanne (EPFL), Switzerland, in 1981 and 1984, respectively.

From 1985 to 1997 , he worked as a Scientist with the National Institutes of Health, Bethesda, MD. $\mathrm{He}$ is now Professor and Director of the Biomedical Imaging Group at the EPFL. His main research area is biomedical image processing. He has a strong interest in sampling theories, multiresolution algorithms, wavelets, and the use of splines for image processing. He is the author of over 120 published journal papers in these areas.

Dr. Unser serves as regular Chair for SPIE's Conference on Wavelets, which has been held annually since 1993. He was General Co-Chair for the First IEEE International Symposium on Biomedical Imaging (ISBI 2002), which was held in Washington, DC, July 7-10, 2002. He also chairs the newly created technical committee of the signal processing society on Bio Imaging and Signal Processing (BISP). He received the 1995 and 2003 Best Paper Awards and the 2000 Magazine Award from the IEEE Signal Processing Society. He is the Associate Editor-in-Chief of the IEEE TRANSACTIONS ON MEDICAL IMAGING and the Editor-in-Chief of the Wavelet Digest, the electronic newsletter of the wavelet community. 